\title{
Use of Targeted SERS Nanosensors in Whole Cell pH Analysis
}

\author{
K. L. Nowak-Lovato* and K. D. Rector** \\ *Bioscience Division, Los Alamos National Laboratory, MS M888, Los Alamos NM 87567 \\ **Chemistry Division, Los Alamos National Laboratory, MS J567, Los Alamos NM 87567
}

The ability to develop a gold-core, silver-shell surface-enhanced Raman Scattering (SERS) nanosensor that has both a targeting component as well as a sensing component has been reported.[1] These targeted nanosensors have shown that they are capable of live cell interrogation of chemical environment while localized to specific cellular pathways based on the ligands chosen for the particle surface, see Figure 1.

The nanosensors is sensitive to $\mathrm{pH}$ changes in the endocytic vesicle within the $\mathrm{pH}$ range of 4.5-7.5 by 4-mercaptopyridine (4-MPy) adsorbed to the particle surface. The $\mathrm{pH}$ dependence of the SERS spectrum of 4-MPy is shown in Figure 2. Vibrational modes at frequencies of $1580 \mathrm{~cm}^{-1}$ and 1612 $\mathrm{cm}^{-1}$ display these proportional ratiometric effects dependent upon $\mathrm{pH}$.[2] These vibrational modes are based on protonation and deprotonation of the ring $\mathrm{N}$ atom in the 4-MPy molecule. The sensing component of the nanosensor is calibrated with in vivo measurements of rat basophil leukemia (RBL-2H3) cells, in standard buffer solutions using the ionophore nigericin, which serves to equilibrate the external $\left[\mathrm{H}^{+}\right]$concentration with that of the cell compartments.

The nanosensor is capable of targeting through the FceRI receptor-mediated endocytic pathway due to the adsorption of 2,4- $\varepsilon$-dinitrophenol-L-lysine (DNP) ligands on the nanoparticle surface. The targeting and sensing moieties do not significantly interfere with each other's function. When these targeted nanosensors are delivered into live cells, whole cell, time lapse, $\mathrm{pH}$ maps can be generated, and these maps can be overlaid with bright field images of the cell, see Figure 3 for an example at $\sim 20$ minutes after nanosensor delivery.

The number of pixels seen in the time lapse imagery increase over the first forty-five minutes of experiments at $25^{\circ} \mathrm{C}$ and decrease over the remaining 45 minutes. The increase in number of pixels seen over time can be conclusively described as more nanosensors being internalized into the cells. However the decrease in number of pixels seen can either be attributed to elimination or aggregation of nanosensors within the same pixel volume. Comparison of integrated intensities of nanosensors over time and space indicated that most of the decrease in pixels seen is from elimination. Further, it would be ideal to be able to more specifically quantitate the number of nanosenors within each endosome compartment so that endosome-specific information can be attributed, or that single endosome compartments can be followed over space and time. The $\mathrm{pH}$ dependence of the integrated SERS $1580 \mathrm{~cm}^{-1}$ signal has been plotted and normalized to total pyridine signal and averaged over time has been determined, which is shown in Figure 4 . The $\mathrm{pH}$ dependence shows a near-linear trend that the integrated SERS signal increases with increasing acidity by approximately $33 \%$ when changed from 7-7.5 range to 4.5-5.0 range.

[1] K.L. Nowak-Lovato and K.D.Rector “Applied Spectroscopy accepted (April, 2009)

[2]Jiawen $\mathrm{Hu}$, Bing Zhao, Weiqing Xu et al., Spectrochimica acta. Part A, Molecular and biomolecular spectroscopy 58A (13), 2827 (2002). 


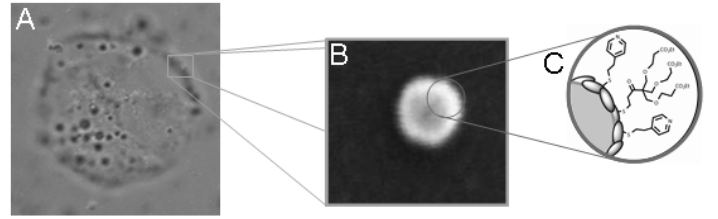

Figure 1. Live cells under stimuli (a) can be interrogated by targeted nanosensors (b) which have both sensing and targeting ligands on the surface (c).

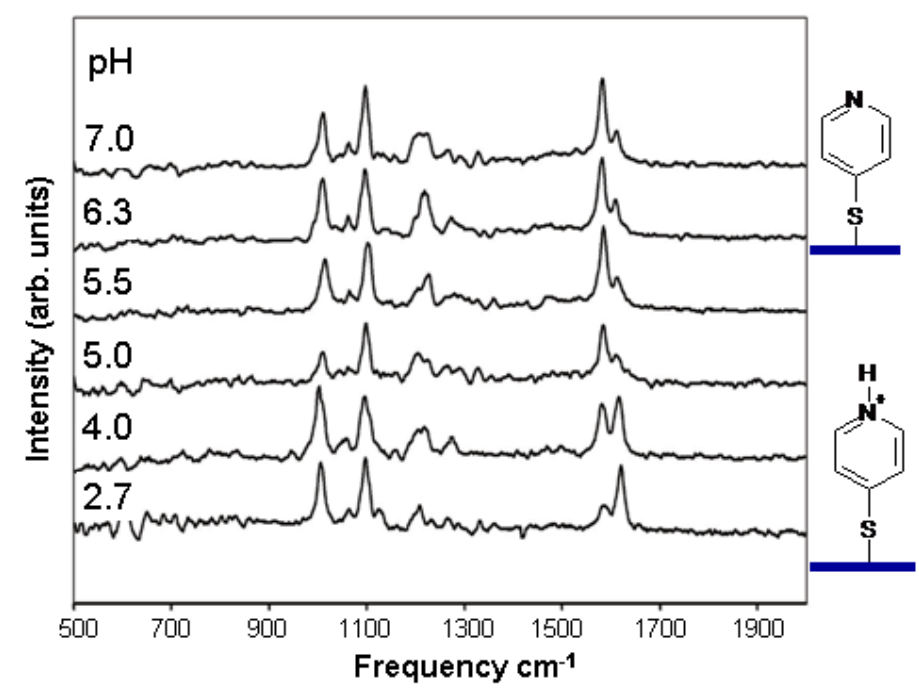

Figure 2. $\mathrm{pH}$-dependent SERS spectra of 4-MPy. Protonation of the pyridine with increasing $\mathrm{pH}$ cause shift of $1580 \mathrm{~cm}^{-1}$ peak to the $1612 \mathrm{~cm}^{-1}$ peak.

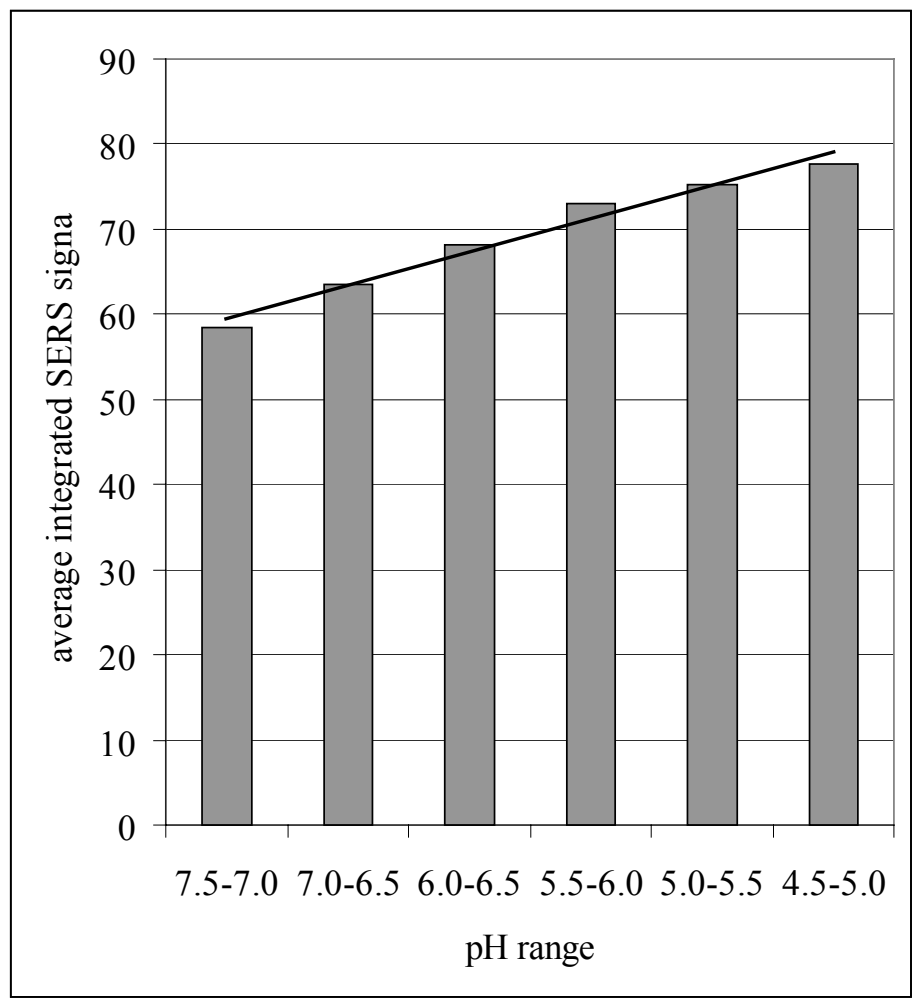

Figure 4. Integrated SERS signal in the 1580 range as a function of $\mathrm{pH}$ normalized to total pyridine signal and average over time. Line is linear fit to the data, to guide the eye.

Figure 3 . Targeted nanosensors delivered to live
RBL cell showing $\mathrm{pH}$ distribution of endosomes at $\sim 20 \mathrm{~min}$. 